\title{
Acute Renal Failure in Tetanus: Authors' Reply
}

\author{
Satnam Kaur • Devendra Mishra • Monica Juneja
}

Received: 7 January 2014 / Accepted: 22 January 2014 /Published online: 27 February 2014

(C) Dr. K C Chaudhuri Foundation 2014

To the Editor: We thank the authors for their letter, which has shed additional light on the issues related to management of tetanus.

- The dose of diazepam $(50 \mathrm{mg} / \mathrm{kg} / \mathrm{d})$ used in the patient was not at all "very high", previous experience with such high doses exists, and the same is in use by our tetanus unit [1-3].

- There has been some concern raised about propylene glycol toxicity in patients receiving diazepam and lorazepam. We were aware of this possibility, but as normal serum levels of propylene glycol are not described and also facilities for measurement are not routinely available, confirmation is difficult and it can be considered as one of the diagnostic possibilities in the patient [4]. However, in an observational study on propylene toxicity, $2 / 3 \mathrm{rd}$ of the patients developing this problem were chronic alcohol abusers, thereby raising the possibility that altered metabolic pathways are responsible for the toxicity [4].

- Magnesium sulfate has been suggested to be of benefit in reducing requirement for other drugs to control muscle spasms and cardiovascular instability in Adult patients with tetanus [5]. However, a more recent meta-analysis suggested the need for more controlled trials to assess the effect of magnesium sulphate on reducing autonomic instability, spasms, duration of intensive care and hospital stays and the need for mechanical ventilation [6].

- The slightly increased creatinine in the range of mild acute kidney injury (AKI) could very well be due to almost absent oral intake since the onset of tetanus. Early and

S. Kaur $(\bowtie) \cdot$ D. Mishra $\cdot$ M. Juneja

Department of Pediatrics, Maulana Azad Medical College, 2, BSZ

Marg, Delhi 110 002, India

e-mail: sk_doc@yahoo.co.in aggressive fluid resuscitation to restore renal perfusion and increase urine flow is the standard management and was done in this patient also. Due to the restriction on the word-limit, it is sometimes difficult to provide minute details of the case, except for the most relevant ones.

- The cited 'consensus statement' is infact a 'qualitative' review [7], and the authors state that there is little evidence other than from animal studies, retrospective observational studies, and case series to support the routine use of bicarbonate-containing fluids.

Contributions SK: Drafted the manuscript; DM: Helped in manuscript writing; MJ: Revised the manuscript for important intellectual content. The final manuscript was approved by all the authors. MJ will act as guarantor for this paper.

Conflict of Interest None.

Role of Funding Source None.

\section{References}

1. Kaur S, Mishra D, Juneja M. Acute renal failure in tetanus. Indian J Pediatr. 2013. doi:10.1007/s12098-013-1008-9.

2. Tullu MS, Deshmukh CT, Kamat JR. Experience of pediatric tetanus cases from Mumbai. Indian Pediatr. 2000;37:765-71.

3. Khoo BH, Lee EL, Lam KL. Neonatal tetanus treated with high dose diazepam. Arch Dis Child. 1978;53:737-9.

4. Wilson KC, Reardon C, Theodore AC, Farber HW. Propylene glycol toxicity: A severe iatrogenic illness in ICU patients receiving IV benzodiazepines. Chest. 2005;128:1674-81.

5. Thwaites CL, Yen LM, Loan HT, Thuy TTD, Thwaites GE, Stepniewska K, et al. Magnesium sulphate for treatment of severe tetanus: A randomised controlled trial. Lancet. 2006;368:1436-43.

6. Rodrigo C, Samarakoon L, Fernando SD, Rajapakse S. A metaanalysis of magnesium for tetanus. Anaesthesia. 2012;67:1370-4.

7. Zimmerman JL, Shen MC. Rhabdomyolysis. Chest. 2013;144:1058-65. 